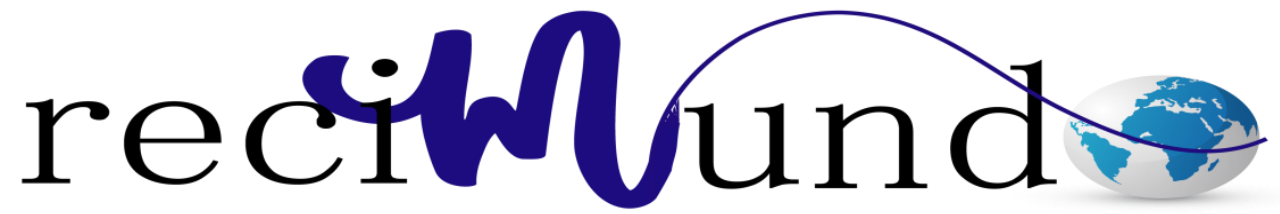

Revista Científica Mundo de la Investigación y el Conocimiento

Jessica Jarrin Yépez ${ }^{\text {a }}$; Jessenia Stefania Cruz Carranza ${ }^{\text {}}$; Anel Montiel Mendoza ${ }^{c}$; Sara Peralta Merelo ${ }^{\text {; }}$ Pavel Carrillo Alvarado ${ }^{\mathrm{e}}$

Condiciones de los recién nacidos de madres con diabetes gestacional en el Hospital

Gineco Obstétrico Enrique C. Sotomayor en septiembre 2015 - agosto 2016

Revista Científica Mundo de la Investigación y el Conocimiento. Vol. 2 núm., especial, mayo, ISSN: 2588-073X, 2018, pp. 956-970

DOI: 10.26820/recimundo/2.esp.2018.956-970

Editorial Saberes del Conocimiento

Recibido: 05/12/2017

Aceptado: $15 / 02 / 2018$

a. Médicos del Hospital Matilde Hidalgo de Procel.

b. Médicos del Hospital Matilde Hidalgo de Procel.

c. Médicos del Hospital Matilde Hidalgo de Procel.

d. Médicos del Hospital Matilde Hidalgo de Procel. 


\section{Condiciones de los recién nacidos de madres con diabetes gestacional en el Hospital Gineco Obstétrico Enrique C. Sotomayor en septiembre 2015 -}

agosto 2016

Vol. 2, núm. Esp., (2018)

Jessica Jarrin Yépez; Jessenia Stefania Cruz Carranza; Anel Montiel Mendoza; Sara Peralta Merelo; Pavel Carrillo Alvarado

\section{RESUMEN}

La presente investigación tiene como objetivo principal, determinar las condiciones de los recién nacidos de madres con diabetes gestacional atendidas en el hospital Gineco Obstétrico Enrique C. Sotomayor, durante el periodo de septiembre del 2015 hasta agosto del 2016. . La documentación percibida abarca variables relacionadas con la edad, paridad, tipo de parto, diabetes gestacional, entre otros, se realizó un estudio retrospectivo, descriptivo, no experimental de corte transversal, con enfoque cuantitativo, la muestra es igual al universo con un total de 64 recién nacidos ya que cumplen con los criterios de inclusión y exclusión , la recolección de datos se hizo mediante la revisión de historias clínicas ; obteniendo como resultado lo siguiente: el sexo de los recién nacidos predominante es el masculino con un 64\%, el 39\% de los neonatos nacieron con un peso de 2001 a 3000 gramos seguido de un 30\% nacidos con un peso de 3001 a 4000 gramos, la talla predominante es de 41 a $50 \mathrm{~cm}$ en un $75 \%$, el $89 \%$ nace con una edad gestacional estimada de 31 a 41 semanas y con un apgar de 8 a 9 en un $31 \%$.

Palabras Clave: Diabetes gestacional, apgar, neonato, edad gestacional. 


\section{Condiciones de los recién nacidos de madres con diabetes gestacional en el Hospital Gineco Obstétrico Enrique C. Sotomayor en septiembre 2015 - agosto 2016}

Vol. 2, núm. Esp., (2018)

Jessica Jarrin Yépez; Jessenia Stefania Cruz Carranza; Anel Montiel Mendoza; Sara Peralta Merelo; Pavel Carrillo Alvarado

\section{ABSTRACT}

The present investigation has as main objective, to determine the conditions of the neonates of mothers with gestational diabetes attended at the Hospital Gineco Obstetric Enrique C. Sotomayor, during the period from September 2015 to August 2016.. Perceived documentation includes variables related to age, parity, type of delivery, gestational diabetes, among others, a retrospective, descriptive, non-experimental cross-sectional study was conducted with a quantitative approach, the sample is equal to the universe with a total of 64 newborns as they met the inclusion and exclusion criteria, data collection was done by reviewing medical records; Resulting in the following: the sex of the predominant newborns is the male with $64 \%, 39 \%$ of the neonates were born with a weight from 2001 to 3000 grams followed by $30 \%$ born with a weight of 3001 to 4000 grams, The predominant size is from 41 to $50 \mathrm{~cm}$ in $75 \%, 89 \%$ is born with an estimated gestational age of 31 to 41 weeks and with an apgar of 8 to 9 in $31 \%$.

Keywords: Gestational Diabetes, Apgar, Neonate, Gestational Age. 


\section{Condiciones de los recién nacidos de madres con diabetes gestacional en el Hospital Gineco Obstétrico Enrique C. Sotomayor en septiembre 2015 -}

agosto 2016

Vol. 2, núm. Esp., (2018)

Jessica Jarrin Yépez; Jessenia Stefania Cruz Carranza; Anel Montiel Mendoza; Sara Peralta Merelo; Pavel Carrillo Alvarado

\section{Introducción.}

La diabetes gestacional es la que se reconoce por primera vez durante la gestación se presenta como una complicación afectando al 7\% de las gestantes, se relaciona con un nivel alto de azúcar en la sangre, no presenta ni signos ni síntomas solo complicaciones, este tipo de diabetes es el que se presenta aproximadamente en el $90 \%$ de las gestantes diabéticas ${ }^{1-5}$.

En caso de diabetes gestacional no controlada existe la posibilidad de que el recién nacido tenga altos niveles de azúcar en su sangre afectando tanto a la madre como al producto. Existen casos de niños con macrosomia y recién nacidos pre términos, y durante el parto pueden aparecer complicaciones como traumatismos, fracturas de clavícula, parálisis braquial. "Él bebe al nacer está más destinado a tener periodos de azúcar en la sangre en el nacimiento y en los posteriores días donde se prevé defectos congénitos"3-7.

De acuerdo a estadísticas oficiales del Instituto Ecuatoriano de Estadística y Censos, señala que la diabetes gestacional ocupó en el año 2009, un sexto puesto como los motivos de morbimortalidad materno fetal. Detalla además que en el año 2012, el sobrepeso de un niño al nacer se ubica en los 10 primeros motivos de morbilidad infantil en el Ecuador ${ }^{2,8}$.

El incremento prevalente de la diabetes, diagnosticado a mujeres en edad más joven, favorece el testimonio de diabetes gestacional debido a los cambios fisiológicos que da el proceso del embarazo, dificultando el control y se asocian a la morbilidad y mortalidad perinatal $^{9-13}$. 


\section{Condiciones de los recién nacidos de madres con diabetes gestacional en el Hospital Gineco Obstétrico Enrique C. Sotomayor en septiembre 2015 - agosto 2016}

Vol. 2, núm. Esp., (2018)

Jessica Jarrin Yépez; Jessenia Stefania Cruz Carranza; Anel Montiel Mendoza; Sara Peralta Merelo; Pavel Carrillo Alvarado

Los neonatos, que son los niños de las madres con diabetes (HMD) están en constante aumento. La diabetes gestacional es la encargada de alterar los sistemas fisiológicos en el feto, al no establecer un adecuado control de la glucemia desde el inicio del embarazo, afectando a la organogénesis, así mismo por el control tardío relacionado con la parte corporal, vinculando al feto macrosomía y las patologías perinatales ${ }^{13-15}$.

No se ha encontrado mecanismo que permita conocer las alteraciones en el feto, mucho menos en el recién nacido que es hijo de una madre con diabetes. La diabetes gestacional cuando no existe control o chequeo médico crea una compensación adversa durante el embarazo que podría perjudicar tanto a la madre como al feto.

En el feto, la insulina actúa como una hormona anabólica primaria de crecimiento fetal y desarrollo, ocasiona macrosomía y visceromegalia a nivel cardiaco y hepático. Cuando hay un exceso de sustrato (glucosa), se produce aumento de la síntesis grasa, y esta se deposita en los órganos antes mencionados, principalmente en el tercer trimestre de la gestación.

La Diabetes Gestacional durante el embarazo es un factor de riesgo, para el desarrollo de complicaciones en la madre y en el feto, siendo en las madres infecciones urinarias, candidiasis vaginal, poli hidramnios, estados hipertensivos del embarazo prematuridad. En el feto y neonato se forman malformaciones y/o abortos, crecimiento intrauterino retardado (CIR), y vasculopatía materna secundaria a diabetes, además en lo que a hiperinsulinismo fetal se refiere aparecen Macrosomía (distocias, traumatismo obstétrico y aumento de la tasa de cesáreas), riesgo de Pérdida de Bienestar Fetal ante o in6traparto, Miocardiopatía hipertrófica e inmadurez 


\section{Condiciones de los recién nacidos de madres con diabetes gestacional en el Hospital Gineco Obstétrico Enrique C. Sotomayor en septiembre 2015 -}

agosto 2016

Vol. 2, núm. Esp., (2018)

Jessica Jarrin Yépez; Jessenia Stefania Cruz Carranza; Anel Montiel Mendoza; Sara Peralta Merelo; Pavel Carrillo Alvarado

fetal que puede manifestarse como síndrome de distréss respiratorio, o alteraciones metabólicas ${ }^{10-15}$.

El objetivo de la presente investigación es determinar las condiciones de los recién nacidos de madres con diabetes gestacional, mediante la revisión de las historias clínicas de las usuarias atendidas en el hospital Gineco Obstétrico Enrique C. Sotomayor.

\section{Metodología.}

Materiales y métodos

Localización

El presente estudio se realizó en el área de pos parto inmediato del Hospital Gineco- Obstétrico "Enrique C. Sotomayor" de la Ciudad de Guayaquil en el centro de la ciudad en las calles Pedro Pablo Gómez y 6 de marzo, la perspectiva de áreas dedicadas a los problemas relacionados a la salud, en el centro con afluencia de personas de todo el territorio ecuatoriano.

Caracterización de la zona de trabajo

El lugar donde se encuentra el hospital gineco-obstétrico enrique c. Sotomayor es de fácil acceso ya que se encuentra en la parte principal y un poco conflictiva.

Período de Investigación 


\section{Condiciones de los recién nacidos de madres con diabetes gestacional en el Hospital Gineco Obstétrico Enrique C. Sotomayor en septiembre 2015 - agosto 2016}

Vol. 2, núm. Esp., (2018)

Jessica Jarrin Yépez; Jessenia Stefania Cruz Carranza; Anel Montiel Mendoza; Sara Peralta Merelo; Pavel Carrillo Alvarado

La investigación se la realiza en el área de estadísticas y censo del hospital "Enrique C.

Sotomayor" en el periodo de septiembre 2015- septiembre 2016. La documentación percibida abarca variables relacionadas con la edad, paridad, tipo de parto, diabetes gestacional, entre otros. La investigación además cuenta con información proporcionada por especialista en el tema, doctores que por años entregaron su vida al desarrollo de la salud materna.

\section{Recursos Físicos}

Los recursos físicos abarcan la información documentada en el historial clínico en el área de estadísticas del Hospital "Enrique C. Sotomayor", además de materiales de oficina, y papelería en general que son utilizados en revisiones e investigaciones, se utiliza además laptop, teléfono celular, Pen-drive, impresora, hojas blancas, carpetas, lápices, bolígrafos, permiso de la institución para realizar el trabajo de investigación, documentación por parte de la Escuela de Obstetricia para realizar el trabajo de investigación.

Universo y muestra

Universo

El universo lo constituye 64 recién nacidos de madres con diabetes gestacional.

Muestra

La muestra es igual que el universo 64 recién nacidos de madres con diabetes gestacional que cumplen con los criterios de inclusión y exclusión. 


\section{Condiciones de los recién nacidos de madres con diabetes gestacional en el Hospital Gineco Obstétrico Enrique C. Sotomayor en septiembre 2015 - \\ agosto 2016}

Vol. 2, núm. Esp., (2018)

Jessica Jarrin Yépez; Jessenia Stefania Cruz Carranza; Anel Montiel Mendoza; Sara Peralta Merelo; Pavel Carrillo Alvarado

Método

Tipo de investigación

El siguiente trabajo es de tipo descriptivo y retrospectivo

Diseño de la investigación

No experimental y de corte transversal.

Procedimiento de la investigación

Recopilar la información en base a las historias clínicas seleccionadas sobre el tema a realice, obtener los respectivos permisos a la institución donde realizaremos el trabajo de investigación.

Averiguar los datos en el departamento de estadística madres diabetes gestacional y de recién nacido, darle a conocer la actividad que realizaremos y obtener su consentimiento.

Operacionalización de instrumentos

Los instrumentos utilizados son las fichas clínicas de las pacientes con diabetes gestacional atendidas en el área de parto del hospital E. C. Sotomayor con diagnóstico de azúcar elevada en la sangre, analizada y diagnosticada por los internos y enfermeras que atiende a los pacientes. 


\section{Condiciones de los recién nacidos de madres con diabetes gestacional en el Hospital Gineco Obstétrico Enrique C. Sotomayor en septiembre 2015 - agosto 2016}

Vol. 2, núm. Esp., (2018)

Jessica Jarrin Yépez; Jessenia Stefania Cruz Carranza; Anel Montiel Mendoza; Sara Peralta Merelo; Pavel Carrillo Alvarado

\section{Resultados.}

Tabla $N^{\circ}$ 1.- Complicaciones de los recién nacidos de acuerdo al sexo

\begin{tabular}{lrr}
\hline SEXO RN & RESULTADOS & \multicolumn{2}{c}{ PORCENTAJE } \\
\hline MASCULINO & 41 & $64 \%$ \\
FEMENINO & 18 & $34 \%$ \\
NEONATOS QUE & & \\
NACIERON MUERTOS & 5 & $2 \%$ \\
MASCULINO & 64 & $100 \%$ \\
TOTAL & &
\end{tabular}

Se determinó en este grafico el mayor porcentaje en los recién nacidos del sexo masculino con un porcentaje del 64\%. Los cual son los que presenta mayor patología.

Tabla $N^{\circ}$ 2.- Edad de las pacientes con diabetes Gestacional

\begin{tabular}{lll}
\hline Edad & descripción & Porcentaje \\
\hline 12 a 18 & 12 & $19 \%$ \\
18 a 26 & 16 & $25 \%$ \\
26 a 34 & 24 & $38 \%$ \\
34 a 40 & 12 & $19 \%$ \\
& 64 & $100 \%$ \\
\hline
\end{tabular}

El 25\% de las personas embarazadas que asisten a la Maternidad Enrique C. Sotomayor tiene una edad que fluctúa entre 18 a 26 años y el $38 \%$ de 26 a 34 años, siendo en esta edad donde se presenta un mayor número de inconvenientes en pacientes con diabetes gestacional por falta de control pre natal y esquemas de nutrición adecuados. 


\section{Condiciones de los recién nacidos de madres con diabetes gestacional en el Hospital Gineco Obstétrico Enrique C. Sotomayor en septiembre 2015 - agosto 2016}

Vol. 2, núm. Esp., (2018)

Jessica Jarrin Yépez; Jessenia Stefania Cruz Carranza; Anel Montiel Mendoza; Sara Peralta Merelo; Pavel Carrillo Alvarado

\section{Tabla $N^{\circ}$ 3.- Paridad de las pacientes}

\begin{tabular}{lcc}
\hline Paridad & Frecuencia & porcentaje \\
\hline Primigesta & 14 & $22 \%$ \\
Segundigesta 1-2 hijos & 32 & $50 \%$ \\
Mutiparidad 3-4 hijos & 15 & $23 \%$ \\
Gran multiparidad 5 a más hijos & 3 & $5 \%$ \\
& 64 & $100 \%$ \\
\hline
\end{tabular}

Los pacientes atendidos en el hospital Maternidad Enrique C. Sotomayor son aquellos que de una u otra forma ya han tenido partos normales o cesarías, siendo el $50 \%$ de las presentadas que ya tienen antecedente de embarazos, y un $22 \%$ son primigesta, donde es aquí en su mayoría donde aparece el problema discernido referente a la diabetes gestacional.

Tabla $N^{\circ}$ 4.- Intervención de las pacientes

\begin{tabular}{lcc}
\hline Intervención & Frecuencia & porcentaje \\
\hline Parto vaginal & 43 & $67 \%$ \\
Parto por cesarea & 21 & $33 \%$ \\
& 64 & $100 \%$ \\
\hline
\end{tabular}

El 67\% de las embarazadas que ingresan al hospital Maternidad Enrique C. Sotomayor tiene un parto normal, sin embargo el restante $33 \%$ reflejada en una muestra de sesenta y cuatro persona presenta un parto con cesaría. Esto implica que los problemas de embarazo gestacional 


\section{Condiciones de los recién nacidos de madres con diabetes gestacional en el Hospital Gineco Obstétrico Enrique C. Sotomayor en septiembre 2015 - agosto 2016}

Vol. 2, núm. Esp., (2018)

Jessica Jarrin Yépez; Jessenia Stefania Cruz Carranza; Anel Montiel Mendoza; Sara Peralta Merelo; Pavel Carrillo Alvarado

se presentan en ambas situaciones de parto., por lo que no existe un adecuado manejo de la alimentación y cuidado en los primeros meses del embarazo.

Tabla $N^{\circ}$ 5.- Determinar el peso de los recién nacidos

\begin{tabular}{lcc}
\hline PESO RN & RESULTADOS & PORCENTAJE \\
\hline $1000-2000$ & 11 & $17 \%$ \\
$2001-3000$ & 25 & $39 \%$ \\
$3001-4000$ & 19 & $30 \%$ \\
$4001-5000$ & 4 & $6 \%$ \\
Neonatos muertos 1000-2000 & 5 & $8 \%$ \\
TOTAL & 64 & $100 \%$ \\
\hline
\end{tabular}

En este gráfico podemos identificar los peso de los recién que el mayor porcentaje lo tiene el peso de 2001 - 3000 con un 39\% siguiéndole los de $3001-4000$.

Tabla $N^{\circ}$ 6.- Determinar las tallas del recién nacido

\begin{tabular}{lcc}
\hline TALLA RN & 2015 & PORCENTAJE \\
\hline $30-40$ & 7 & $8 \%$ \\
$41-50$ & 47 & $75 \%$ \\
$51-60$ & 5 & $8 \%$ \\
Neonatos muertos talla $30-40$ & 5 & $9 \%$ \\
TOTAL & 64 & $100 \%$ \\
\hline
\end{tabular}

En el gráfico de las talla de los recién nacidos se puede que la talla que más predomina en los hijos de madres diabéticas con un 75\% es de la talla 41-50. 
Condiciones de los recién nacidos de madres con diabetes gestacional en el Hospital Gineco Obstétrico Enrique C. Sotomayor en septiembre 2015 agosto 2016

Vol. 2, núm. Esp., (2018) Jessica Jarrin Yépez; Jessenia Stefania Cruz Carranza; Anel Montiel Mendoza; Sara Peralta Merelo; Pavel Carrillo Alvarado

\section{Tabla $N^{\circ}$ 7.- Determinar la edad gestacional de los recién nacidos}

\begin{tabular}{lrr}
\hline EDAD GESTACIONAL & RESULTADOS & \multicolumn{2}{c}{ PORCENTAJE } \\
\hline 20-30 SG & 7 & $8 \%$ \\
31-37 SG & 52 & $89 \%$ \\
Nacieron muertos 20-30 SG & 5 & $3 \%$ \\
TOTAL & 64 & $100 \%$ \\
\hline
\end{tabular}

Se identifica en el grafico la edad gestacional de los recién nacidos predomina con un 89\% las semanas desde 31 a 41 de gestación.

Tabla $N^{\circ}$ 8.- Determinar el Apgar de los recién nacidos

\begin{tabular}{lrr}
\hline APGAR & RESULTADOS & \multicolumn{2}{c}{ PORCENTAJE } \\
\hline $4 / 5$ & 1 & $2 \%$ \\
$5 / 6$ & 1 & $2 \%$ \\
$6 / 7$ & 10 & $14 \%$ \\
$5 / 6$ & 7 & $11 \%$ \\
$7 / 7$ & 2 & $3 \%$ \\
$7 / 8$ & 4 & $6 \%$ \\
$8 / 8$ & 5 & $8 \%$ \\
$8 / 9$ & 20 & $31 \%$ \\
$9 / 9$ & 5 & $8 \%$ \\
$9 / 10$ & 4 & $6 \%$ \\
NACIERON MUERTOS & 5 & $9 \%$ \\
TOTAL & 64 & $100 \%$ \\
\hline
\end{tabular}




\section{Condiciones de los recién nacidos de madres con diabetes gestacional en el Hospital Gineco Obstétrico Enrique C. Sotomayor en septiembre 2015 - agosto 2016}

Vol. 2, núm. Esp., (2018)

Jessica Jarrin Yépez; Jessenia Stefania Cruz Carranza; Anel Montiel Mendoza; Sara Peralta Merelo; Pavel Carrillo Alvarado

En el gráfico se determina los apgar de los recién el mayor porcentaje es $31 \%$ Rn con apgar $8 / 9$ a pesar de que vienen con un distres respiratorio.

\section{Conclusiones.}

Según la tabulación de los datos de las historias clínicas se determinó que los recién nacidos de madres con diabetes gestacional en un $75 \%$ tienen una talla de 41 a $50 \mathrm{~cm}$, la edad gestacional más frecuente de nacimiento es entre las 31 a 41 semanas que corresponde a un $89 \%$ de neonatos.

El sexo predominante fue el masculino con un número de 41 neonatos que corresponde al $64 \%$ del grupo en estudio.

El número de productos en estudio que nacieron por cesárea fue de 21 neonatos que corresponden al $33 \%$ mientras que por parto vaginal nacieron 43 neonatos que corresponde a un $67 \%$ del total.

Los recién nacidos de madres con diabetes gestacional nacen con un peso mayor de 3000 gramos en un $30 \%$ mientras que el $39 \%$ nacen con un peso de 2001 a 3000 gramos.

La hipótesis se rechaza ya que los recién nacidos que nacen con apgar menor a 5 solo se presentan en un 2\%, mientras que los recién nacidos con apgar de 8 a 9 se encuentran en un $31 \%$. 


\section{Condiciones de los recién nacidos de madres con diabetes gestacional en el Hospital Gineco Obstétrico Enrique C. Sotomayor en septiembre 2015 -}

agosto 2016

Vol. 2, núm. Esp., (2018)

Jessica Jarrin Yépez; Jessenia Stefania Cruz Carranza; Anel Montiel Mendoza; Sara Peralta Merelo; Pavel Carrillo Alvarado

La edad más frecuente de las madres de los neonatos en estudio está comprendida entre 26 a 34 años que representan un porcentaje del $38 \%$, seguido de las edades de 18 a 26 años con un porcentaje del $25 \%$.

Se identificó que las madres de los recién nacidos son mayormente secundigestas en un numero de 32 que representan el $50 \%$ del total de estudio.

Por lo tanto se determina que las condiciones de nacimiento de los neonatos de madres con diabetes gestacional son óptimas en la mayoría de casos dentro de la investigación.

\section{Bibliografía.}

1. McDonald SJ, Middleton P. Efecto del momento de clampeo del cordón umbilical en recién nacidos término sobre los resultados en la madre y el neonato. ; 2012.

2. INEC IndEyC. El embarazo gestacional. Quito Ecuador:; 2015.

3. Begley , Gyte , Murphy DDJMM. Active versus expectant management for women. NY:; 2014.

4. Karlsson H, Pérez SC. Hemorragia postparto. Pamplona:; 2014.

5. Peñafiel J. Complicaciones en el post parto. Quito Ecuador:; 2015.

6. Prendiville JEHREMS. The Bristol third stage trial: active versus physiological management of third stage NY: BMJ, 297 (1988), pp. 1295-1300; 2012.

7. Safe M, Newborn H. Prevention and treatment of postpartum hemorrhage in low-resource settings. International Journal of Gynecology and Obstetrics. NY:; 2012.

8. Ramos BVR. Complicaciones en el paciente con puerperio inmediato atendidas en el servicio de ginecología del hospital provincial general docente RíoBamba Riobamba : Escuela Superior de Politécnica de Chimborazo; 2014. 


\section{Condiciones de los recién nacidos de madres con diabetes gestacional en el Hospital Gineco Obstétrico Enrique C. Sotomayor en septiembre 2015 - agosto 2016}

Vol. 2, núm. Esp., (2018)

Jessica Jarrin Yépez; Jessenia Stefania Cruz Carranza; Anel Montiel Mendoza; Sara Peralta Merelo; Pavel Carrillo Alvarado

9. Lugo SDAM. Caracterización de las pacientes con hemorragia uterina puerperal en las que se utilizó misoprostol. ; 2014.

10. Vélez-Álvarez GA, Agudelo-Jaramillo B. manejo de la hemorragia obstétrica. Obtetricia y Ginecologia. 2016;: p. 23.

11. Ramírez J. Hemoragias Postparto. Valencia; 2014.

12. Calle A, Barrera M, Alexander G. Diagnóstico y manejo de la hemorragia posparto. Revista PeRuana de GinecoloGía y obstetRicia. 2013;: p. 234.

13. Palomo TJB. INCIDENCIA DE LA HEMORRAGIA POSTPARTO Guatemala : UNIVERSIDAD DE SAN CARLOS DE GUATEMALA; 2014.

14. Weisbrod A, Sheppard F, Chernofsky M, Blankenship C, Frederick G. Manejo emergente de la hemorragia postparto para el tratamiento general y agudo cirujano de cuidado. Diario Mundial de Cirugía de Emergencia. 2015;: p. 1-12.

15. Rodriguez SR. Atonía uterina y factores asociados. Lima; 2011. 